\title{
Disordering and reordering of mechanically milled superlattice alloys
}

\author{
ROBERT W CAHN \\ Department of Materials Science and Metallurgy, University of Cambridge, Pembroke Street, Cambridge CB2 3QZ, \\ England
}

\begin{abstract}
Highenergy mechanical working of ordered alloys has the twin effects of refining the grain size to the nano-range, and reducing (or even eliminating) the long-range order (LRO). In this way, states of order can be achieved that cannot be obtained by heat-treatment. Subsequent annealing will re-establish the order. Some experiments in this field, notably with $\mathrm{Ni}_{3} \mathrm{Al}$, will be outlined.
\end{abstract}

Keywords. Order-disorder transformations; mechanical milling.

\section{Introduction}

When a metallic solid solution with long-range atomic order (LRO) is subjected to high-energy irradiation or mechanical working, it is liable to begin disordering at the same time as it becomes nanocrystalline, and if the treatment is continued long enough, the order-i.e. the superlattice-may disappear entirely; on further working, in some cases, the alloy even becomes amorphous. The preferred method of high-energy working is by some variant of ball-milling, a small amount of powdered alloy being ground between a number of large steel balls subject to some form of vibration. Much of the extensive research in this area during the past 10 years has focussed on such amorphization and its relation to the grain size and reduced long-range order, but here I wish to focus just on the initial stage, the disordering itself, and report on a body of collaborative work done by an interuniversity group in Europe in recent years.

There are quite a number of overviews of the abovementioned processes. A general overview of the metastable states attained through electron or heavy-particle irradiation of ordered alloys and a listing of further relevant specialized reviews can be found in a book chapter on Metastable States of Alloys (Cahn and Greer 1996). The mechanics and physics of mechanical milling and its consequences have been surveyed by Koch (1991) and Suryanarayana (1999). The specific issue of mechanically-driven disordering has been brilliantly surveyed by Bakker et al (1995).

It is well established (see references in Cahn and Greer 1996, and a summary in the review by Bakker et al 1995, p. 190) that a critical degree of disordering must be achieved before amorphization is possible in principle. The reduction of grain size into the nanocrystalline range seems also to be vital: in Bakker's words, 'Part of the increase of the enthalpy (caused by mechanical milling) will reside in the grain boundaries, which constitute an appreciable fraction of the material volume. Further enthalpy increase may be due to dislocations, vacancies and interstitials, although atomic disordering and grain-boundary energy are generally considered as the main source of enthalpy storage. A criterion for amorphization is that the total stored energy should exceed the energy difference between the compound and the amorphous state'. This criterion has been worked out in detail by Beke et al (1991). Others have inclined to the view that a critical vacancy concentration is the chief criterion: the jury is still out.

While the conditions for amorphization have been exhaustively analysed, less attention has been paid to the earlier stage: loss of long-range atomic order. It is clear however that the ordering energy (the difference in energy between the fully-ordered and the fullydisordered states of an alloy phase) has a major influence on whether order can be substantially destroyed by mechanical means or by irradiation. This matter was discussed in some detail (mostly with regard to irradiation) by Russell (1985). Thus NiAl, which has a very high ordering energy according to calculations (implying a virtual disordering temperature well over $5000 \mathrm{~K}$, far above the melting temperature), cannot be detectably disordered by either milling or irradiation. $\mathrm{Ni}_{3} \mathrm{Al}$ has a measured virtual disordering temperature of $\approx 1720 \mathrm{~K}$, barely above the melting temperature (Cahn et al 1987); this means that it has a fairly weak ordering energy and it can easily be totally disordered by milling, as we shall see below. AlRu, which has the same simple superlattice as $\mathrm{NiAl}$, is fairly strongly ordered and can accordingly be only partly disordered by milling (Hellstern et al 1989; Bakker et al 1995); the same applies to $\mathrm{CuTi}$ (Seki and Johnson 1990). Two recent studies of the milling of AlFe (again, fairly strongly ordered) have both found that only partial disordering is possible here, down to an $S$ value of 0.25-0.3 (Peng and Collins 1997; Clavaguera-Mora et al 1997). In all these cases, a balance 
is reached between mechanical disordering and simultaneous thermal reordering.

The calculation of ordering energy, from first principles or by CALPHAD methods, is still an imprecise art and this is no doubt as to why the presumed correlation between the ordering energy and the feasibility of mechanical disordering has not yet been analysed in detail.

A further complication arises when the degree of disordering achieved by milling, as assessed by different kinds of physical measurements, gives varying results. Bakker et al (1995) present the case of the phase CoZr: the degree of disordering was estimated from measurements of the intensities of superlattice and fundamental $\mathrm{X}$-ray diffraction (XRD) lines, from lattice parameters, and from magnetization measurements. Bakker believes that the X-ray intensity method is less sensitive than magnetic measurements when the alloy has become nanocrystalline, but in the present research we focus on XRD methods.

Yet another complication arises when alternative modes of disordering, by milling or by heat-treatment, yield different crystal structures, as has been shown for the $\mathrm{L} 2{ }_{1}$-ordered Heusler alloy, $\mathrm{Ni}_{2} \mathrm{MnSn}$, by the use of Mössbauer spectrometry (Le Caër et al 1997). However, the simpler superlattices discussed in this paper do not seem to be subject to this complication.

\section{States of order and lattice parameter}

The historical development of knowledge and understanding concerning superlattices in alloys goes back to XRD experiments on $\mathrm{Cu}_{3} \mathrm{Au}$ by Bain in 1923 and Johansson and Linde in 1925, as set out in my recent essay, 'The place of atomic order in the physics of solids and physical metallurgy' (Cahn 1994), $\mathrm{Cu}_{3} \mathrm{Au}$ and many other ordered alloys studied in the early days, such as $\mathrm{FeCo}$ and $\mathrm{Ni}_{3} \mathrm{Mn}$, are 'reversibly ordered': a term I introduced some years ago, which means that by appropriate quenching from high temperature or slow cooling, various degrees of order can be attained. An important distinction must be made here between the two types of reversibly-ordered alloys, typified by $\mathrm{FeCo}$ and $\mathrm{Cu}_{3} \mathrm{Au}$. Figure 1 shows the variation of the equilibrium Bragg long-range order parameter, $S$, with temperature, for each of these alloys. One of the early triumphs of the statistical mechanics of solids was the interpretation of this difference by Bragg and Williams (1934). In this paper I focus exclusively on the second $\left(\mathrm{Cu}_{3} \mathrm{Au}\right)$ type of relationship, and I draw attention to the fact that, in equilibrium, $S$ can only adopt values in the range 1.0 (perfect order) to 0.8 ; at the critical temperature (here $395^{\circ} \mathrm{C}$ ) there is an abrupt drop of $S$ from 0.8 to zero (no long-range order). For this kind of alloy, states of order between 0 and 0.8 therefore cannot be examined in equilibrium. If, like $\mathrm{Ni}_{3} \mathrm{Al}$, the alloy is 'permanently ordered'-i.e. long-range order remains up to the melting temperature-there is again no way of obtaining degrees of order much less than 1.0 in equilibrium, though very slight reductions of $S$ below 1.0 , as assessed by resistivity (Kozubski and Cadeville 1988) or by $\gamma$-ray diffraction (Bremer et al 1988), can be achieved by heating close to the melting-temperature.

One feature of ordered alloy phases that has been little studied over the years is the relationship between lattice parameter and order parameter, partly because it is often difficult to attain alloys in states of metastable partial order. This consideration was the main reason why I decided a few years ago to launch an investigation into this aspect of $\mathrm{Ni}_{3} \mathrm{Al}$, using mechanical milling as a means of obtaining states of partial long-range order over the entire range of $S$.

In our first report on this research (Gialanella et al 1992a), we examined the rather limited history of research on the relationship between the order and lattice
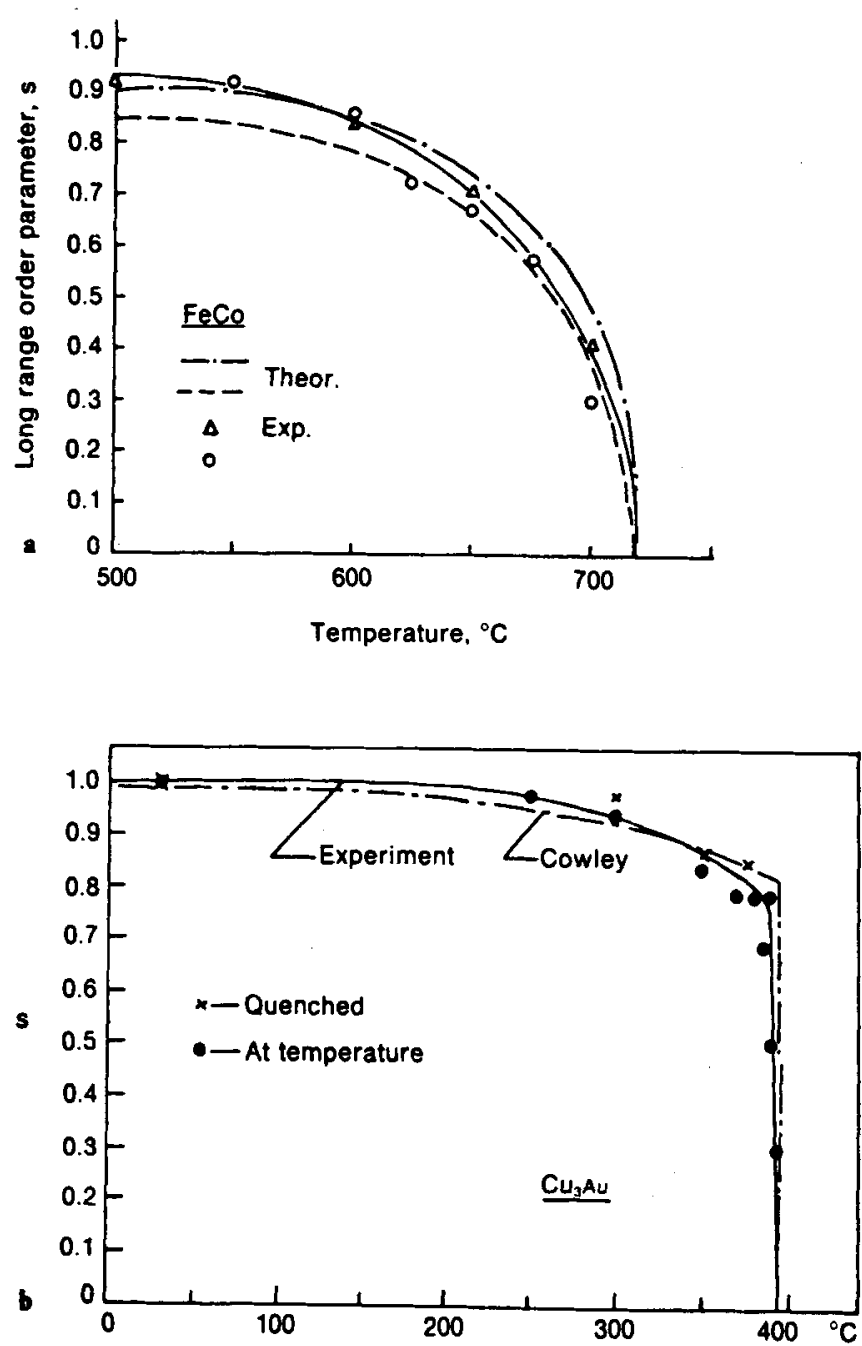

Figure 1. The Bragg order parameter, $S$, as a function of temperature, as measured for (a) $\mathrm{FeCo}$, and (b) $\mathrm{Cu}_{3} \mathrm{Au}$. 
parameters. The lattice parameter increases as the degree of order diminishes, because the constituent atoms are generally of substantially different sizes and such atoms can pack together with less disturbance when they do so in an ordered array. This idea was first put into quantitative form by Betteridge (1949) and later by Dienes (1958). Figure 2, from Betteridge's early paper, shows the lattice parameters of $\mathrm{Cu}_{3} \mathrm{Au}$ equilibrated at different temperatures with some values of the short-range order parameter, $\sigma$, inserted (short-range order persists above the critical temperature). In the figure, the solid line is calculated from Peierls' statistical superlattice theory (Peierls 1936). The fit is satisfactory. The only other measurement for $\mathrm{Cu}_{3} \mathrm{Au}$ was by Feder et al (1958); they showed an approximately linear dependence of lattice parameter on $S$ in the range $0.8-1.0$, but it is difficult to get a clear relationship over such a limited range of $S$.

The study by Feder $e t$ al and by others performed on $\mathrm{Cu}_{3} \mathrm{Au}$, agree on a lattice expansion of $0 \cdot 13-0.16 \%$ between full order and full disorder (attained in all cases by thermal treatment). For other weakly-ordered alloys which are disordered by heat-treatment, the expansion was similar. More strongly-ordered alloys which are disordered (wholly or partly) by milling, the change is

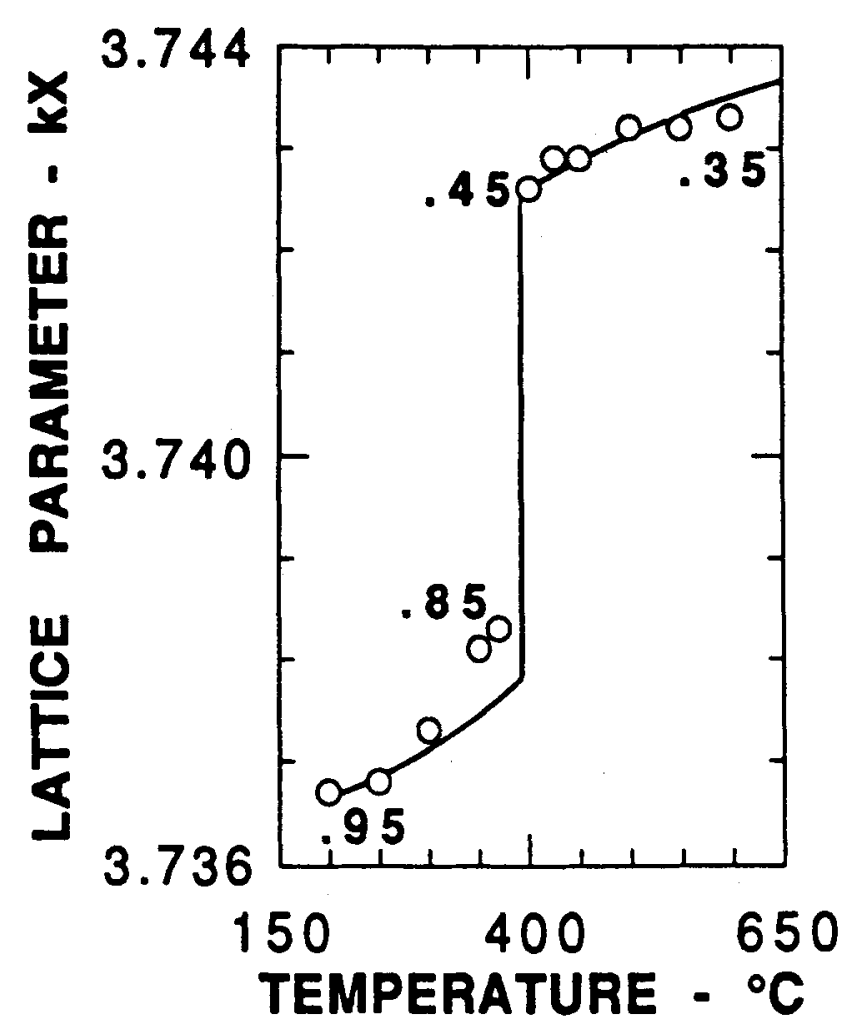

Figure 2. Lattice parameter of $\mathrm{Cu}_{3} \mathrm{Au}$ versus the anneiling temperature (from Betteridge 1949). Full line: calculated from Peierls's theory. Circles: experimental values. Some values of the short-range order (SRO) parameter are indicated. apt to be somewhat larger (e.g. $\approx 0.5 \%$ for partial disordering of AIFe, according to Peng and Collins 1997).

A few measurements of lattice parameter as a function of irradiation time (for $\mathrm{Zr}_{3} \mathrm{Al}$ ) or of milling time (for the A15 compounds $\mathrm{Nb}_{3} \mathrm{Sn}$ and $\mathrm{AuNb}_{3}$, as described by Bakker et al (1995)), were made at about the time we started our investigation, but there was no attempt to determine the $S$ values for the partially-disordered material. An independent study of the mechanicallyinduced disordering of $\mathrm{Ni}_{3} \mathrm{Al}$ (Jang and Koch 1990) was published soon after we started our researches; they went on to study the microhardness of powder particles at various stages of milling. In our work, we examined the nanocrystalline character of the milled powder as well as the state of order.

Very recently, Bakker et al (1997) have again reviewed the role of enthalpy changes in milling-induced structural changes in intermetallics; in this paper he also argues that whereas the lattice parameter should always increase on disordering of 'normal' intermetallics, where disorder is accommodated in the form of antisite atoms, it should decrease for those phases which disorder by creating structural vacancies. However, we are not concerned with such phases in the present paper.

\section{Experimental}

We used boronated hypo-stoichiometric $\mathrm{Ni}_{76 \cdot 6} \mathrm{Al}_{23 \cdot 3}\left(\mathrm{~B}_{0.1}\right)$ powder, in vacuum-atomized form, milled in a planetary ball-mill using tempered chrome-steel balls and vials, all degassed and kept under argon. The lattice parameter particle size and rms strain after milling were determined by standard XRD techniques from measured line angles and integrated intensities and corrected line widths, using monochromated $\mathrm{CuK} \alpha$ radiation; the techniques are described in many texts, e.g. Klug and Alexander (1974). The powder as received was approximately fully ordered $\left(S_{0}\right.$ is assumed $\left.=1\right)$. After milling to the point of complete disordering, powder samples were progressively annealed, either in evacuated tubes or in a differential scanning calorimeter (DSC), to examine the reordering characteristics.

\section{Milled $\mathrm{Ni}_{3} \mathrm{Al}$ powder: observations and discussion}

The long-range order parameter diminished rapidly with continued milling, as shown in figure 3 . The results are roughly consistent with Jang and Koch's (1990) corresponding findings. In another run, the change of lattice parameter, $\Delta a / a$, with milling time was measured (figure 4 ); the wholly unexpected feature here is the continued increase of the lattice parameter long after all long-range order (LRO) had disappeared, according to XRD $(S=0)$. At this stage $\Delta a / a$ is only $\approx 0.25 \%$, while at its peak 
$\Delta a / a$ reaches 4 times this value. We conclude that this additional lattice expansion is due principally, or wholly, to the destruction of short-range order. However, our $\mathrm{X}$-ray findings were called into question by TEM selected-area diffraction patterns from thin foils prepared from milled powders. Powder with $\Delta a / a=0.33 \%$ still showed faint superlattice spots in some areas (none in others), though powders milled for longer times than this one showed no superlattice spots. Thus it seems that XRD, when applied to nanocrystalline powder with the resultant very broad diffraction lines, is not sufficiently sensitive to detect the last traces of LRO. The problem is akin to that relating to $\mathrm{CoZr}$, mentioned in $\S 1$, above. Nevertheless, at least half the total lattice expansion shown in figure 4 must be attributed to something other than the destruction of LRO.

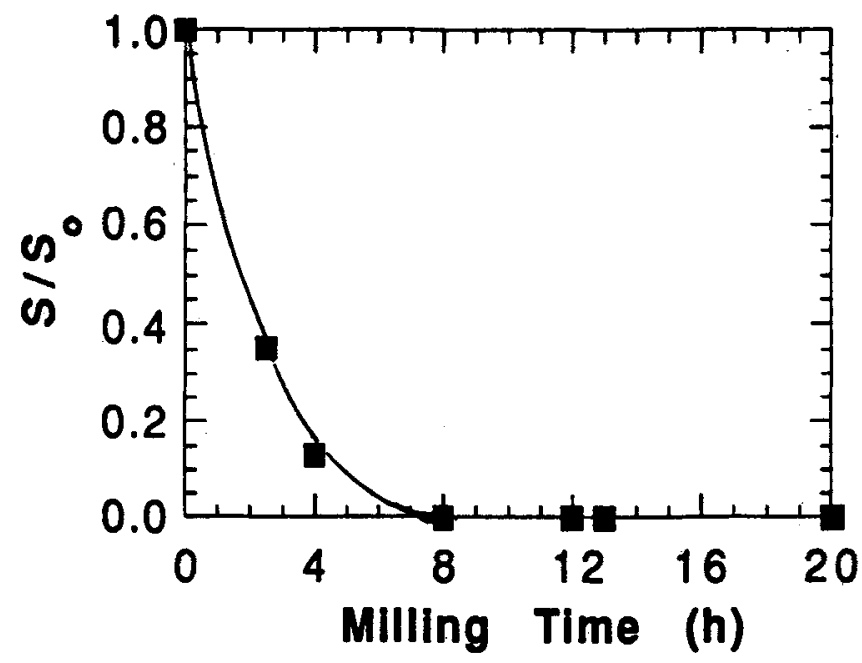

Figure 3. Variation of the reduced long-range order (LRO) parameter, $S / S_{0}$, as a function of the milling time for $\mathrm{Ni}_{3} \mathrm{Al}$ powder.

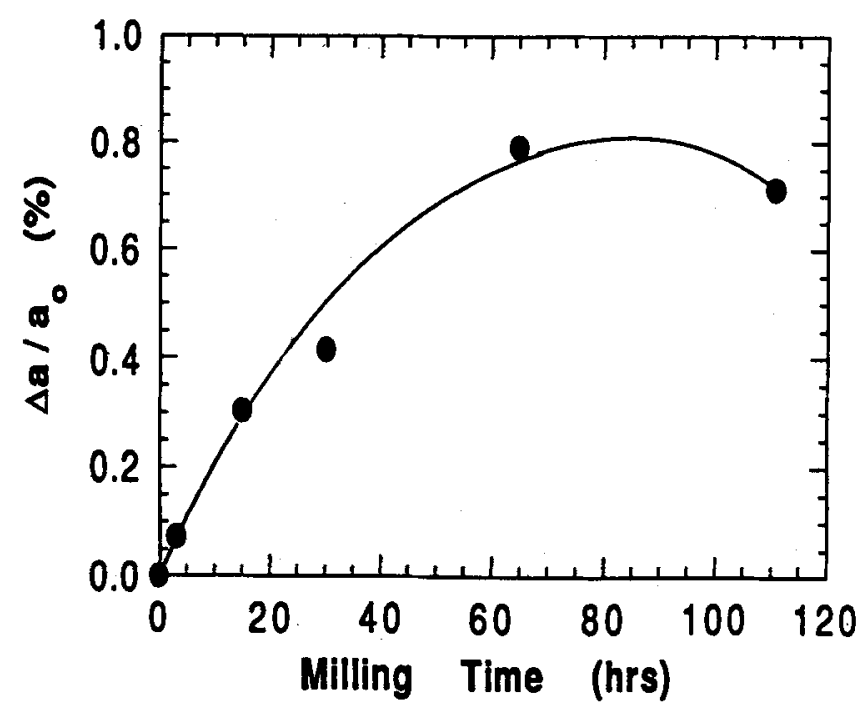

Figure 4. Lattice expansion versus milling time for $\mathrm{Ni}_{3} \mathrm{Al}$ powder.
These early results were determined by Gialanella et al (1992a). More detailed results were published by Gialanella et al (1992b); the most important, in the light of our initial objectives, are those shown in figure 5 . The results here, from a number of runs, relate the measured order parameter (by XRD) to $\Delta a / a$. The straight line is what might have been expected from Betteridge's theory of 1949, which predicted a straight-line relationship between short-range order parameter and lattice parameter, but this graph only shows the part of the expansion which is clearly related to LRO. This relationship, for an alloy with the $\mathrm{Cu}_{3} \mathrm{Au} / \mathrm{Ni}_{3} \mathrm{Al}$ superlattice, could not have been obtained in any other way but that used in this study. Reannealing experiments (Gialanella et al 1992a) proved that the lattice parameter changes were completely reversible and thus contamination by the grinding medium could not have played any part in causing the lattice expansion on grinding.

The last part of the experimental programme was a detailed study of changes associated with reannealing. Full details of this can be found in papers by Gialanella et al (1992b) and Barò et al (1993).

The first point to make is that there appears to be a close correlation between the amount of LRO and the size of the antiphase domains (as estimated from breadth analysis of superlattice lines only) brought about by milling and the subsequent increase caused by reannealing. This is brought out neatly in figure 6 (Gialanella $e t$ al $1992 b$ ), in which are plotted results from a number of reannealing runs of initially completely disordered specimens. Extrapolation of this linear relationship back to $S=0$ gives a domain size of only $10 \AA$; a $10 \AA$ antiphase domain size would be compatible with residual SRO in the heavily milled powder. (At this kind of antiphase domain dimension, the distinction between LRO and SRO becomes fuzzy). The estimated antiphase domain

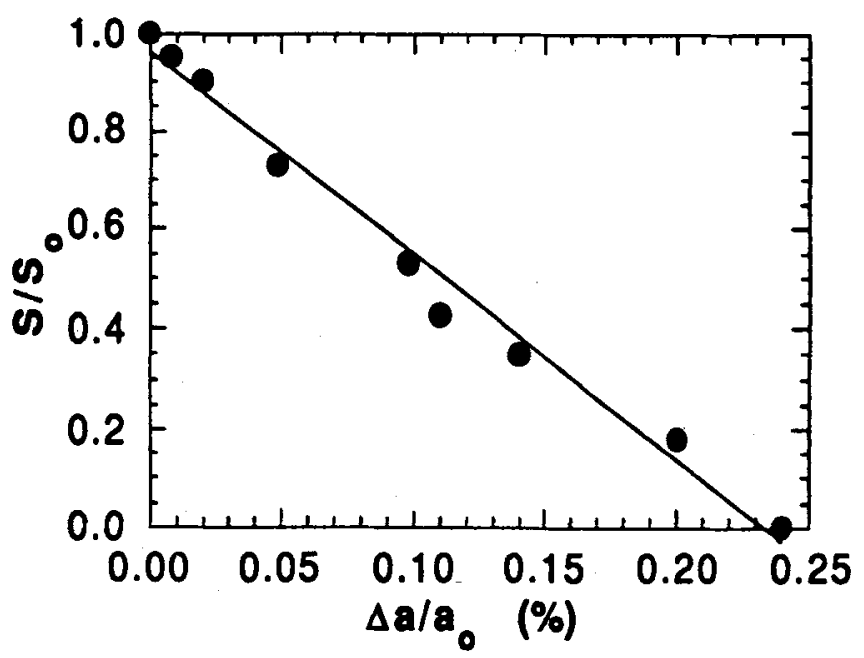

Figure 5. Relationship between lattice expansion and reduced LRO parameter, $S / S_{0}$, in ball-milled $\mathrm{Ni}_{3} \mathrm{Al}$. 


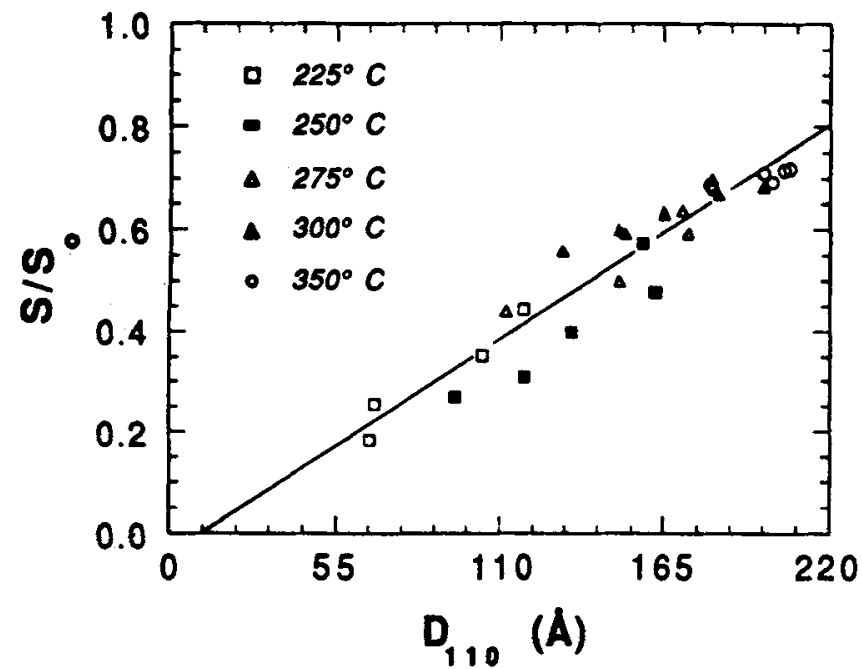

Figure 6. Variation of the reduced LRO parameter versus the average antiphase domain size resulting from isothermal anneals after long-time milling.

size determined by extrapolation back to $S=0$ compares with a measured size of coherently diffracting crystallites (determined from breadth analysis of fundamental diffraction lines) of $\approx 100 \AA$.

A range of DSC experiments, in both continuous-heating and isothermal modes, were done to establish the heat emitted by reestablishment of LRO during annealing. Figure 7 (Barò et al 1993) shows a continuous-heating plot for fully-disordered material (milled for $20 \mathrm{~h}$ ). It can be seen that in addition to the main reordering peak at about $650 \mathrm{~K}$, there is a distinct peak on the lowtemperature shoulder (at about $450 \mathrm{~K}$ ), and a barely detectable peak on the high-temperature shoulder of the main peak (at about $770 \mathrm{~K}$ ). According to experiments done with disordered $\mathrm{Ni}_{3} \mathrm{Al}$ thin films deposited on a cold substrate (Harris et al 1991), the $450 \mathrm{~K}$ peak (which is too low to correspond to any restoration of LRO) can be confidently attributed to the reestablishment of SRO. It is striking that SRO, which is the harder to remove by milling, is also the easier to restore by annealing. The faint high-temperature peak may be associated with the annealing-out of dislocations. In our 1993 paper (Barò et al 1993), a quantitative model for the kinetics of the reestablishment of LRO is successfully fitted to the isothermal observations.

\section{Conclusions}

The use of mechanical milling of $\mathrm{Ni}_{3} \mathrm{Al}$ has allowed the linear relation between LRO parameter, $S$, and lattice parameter, $a$, to be confirmed for the first time over the entire range of $S$. So far as I am aware, no correlation of this kind has been checked for any other alloy. The strong influence of SRO on lattice parameter has also

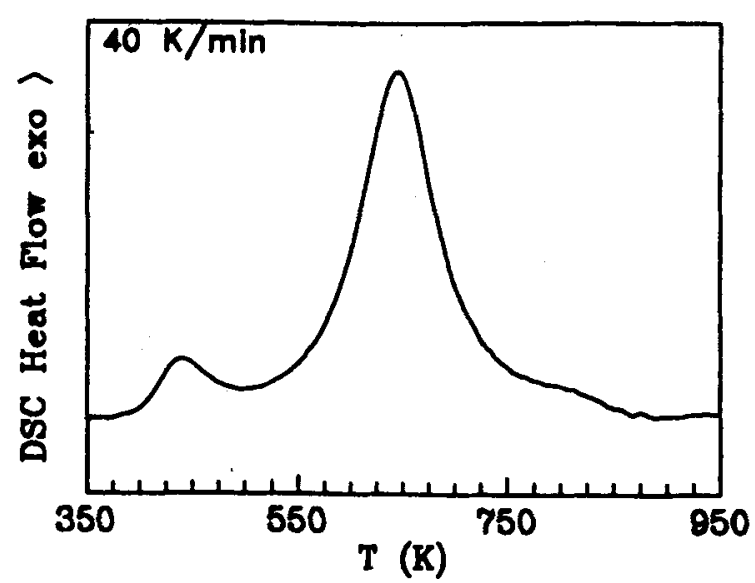

Figure 7. Differential scanning calorimeter record (continuous heating) after $20 \mathrm{~h}$ milling.

been confirmed. Milling has been found to create a nanocrystalline structure with corresponding ultrafine antiphase domain sizes.

The discussion of the correlation between ordering energy and the capacity of a phase to be mechanically disordered entirely, partly or not at all showed that this correlation needs further attention, making use of present-day possibilities of estimating ordering energies either from measured critical temperatures, CALPHAD computations or first-principles calculations.

\section{Acknowledgements}

I am grateful to my collaborators at the Universities of Trento, Italy, and Autonoma de Barcelona, Spain and at the LTPCM-CNRS Laboratory in Grenoble, France for an enjoyable joint research project, and to the Commission of the European Union for financial support.

\section{References}

Bakker H, Zhou G F and Yang H 1995 Prog. Mater. Sci. 39159 Bakker H, Modder I W, Zhou G F and Yang H 1997 Mater. Sci. Forum 235-238 477

Barò M D, Suriñach S, Malagelada J, Clavaguera-Mora M T, Gialanella S and Cahn R W 1993 Acta Metall. Mater. 41 1065

Beke D L, Loeff P I and Bakker H 1991 Acta Metall. Mater. 391259,1267

Betteridge W 1949 J. Inst. Metals 75559

Bragg W L and Williams E J 1934 Proc. R. Soc. London 145 699

Bremer F J, Beyss M and Wenzl H 1988 Phys. Status Solidi (a) 11077

Cahn R W 1994 in Physics of new materials (ed.) F E Fujita (Berlin, Heidelberg: Springer) vol. 27 of Springer Series in Materials Science, p. 179. Second ed., 1998 
Cahn R W and Greer A L 1996 in Physical metallurgy (eds) R W Cahn and P Haasen (Amsterdam: North-Holland) pp. 1747, 1758

Cahn R W, Siemers P A, Geiger J E and Bardhan P 1987 Acta Metall. 352737

Clavaguera-Mora M T, Zhi J, Meyer M, Mendoza-Zelis L, Sanchez F H and Clavaguera N 1997 Mater. Sci. Forum 235-238 541

Dienes G J 1958 Acta Metall. 8278

Feder R, Mooney M and Nowick A S 1958 Acta Metall. 6 266

Gialanella S, Newcomb S B and Cahn R W 1992a in Ordering and disordering in alloys (ed.) A R Yavari (London: Elsevier Applied Science) p. 67

Gialanella S, Cahn R W, Malagelada J, Suriñach S, Barò M D and Yavari A R 1992b in Kinetics of ordering transformations in metals (eds) $\mathrm{H}$ Chen and $\mathrm{V} \mathrm{K}$ Vasudevan (Warrendale, PA: TMS) p. 161

Harris S R, Pearson D H, Garland C M and Fultz B $1991 \mathrm{~J}$. Mater. Res. 6201
Hellstern E, Fecht H J, Fu Z and Johnson W L 1989 J. Appl. Phys. 65305

Jang J S C and Koch C C 1990 J. Mater. Res. 5598

Klug H P and Alexander L E 1974 X-ray diffraction procedures (New York: Wiley) 2nd ed

Koch C C 1991 in Processing of metals and alloys (ed.) R W Cahn (Weinheim: VCH), vol. 15 of Materials Science and Technology p. 193

Kozubski R and Cadeville M S 1988 J. Phys. F18 2569

Le Caër G, Delcroix P, Malaman B, Welter R, Fultz B and Ressouche E 1997 Mater. Sci. Forum 235-238 583

Peierls R 1936 Proc. R. Soc. London Ser. A 166376

Peng L S J and Collins G S 1997 Mater. Sci. Forum 235-238 535

Russell K C 1985 Prog. Mater. Sci. 28229

Seki Y and Johnson W L 1990 in Solid state powder processing (eds) A $\mathrm{H}$ Clauer and $\mathrm{J} \mathrm{J}$ de Barbadillo (Warrendale, PA: TMS) p. 287

Suryanarayana C (ed.) 1999 in Non-equilibrium processing of materials (Oxford: Pergamon Press) (in press) 